\title{
Density functional for van der Waals forces accounts for hydrogen bond in benchmark set of water hexamers
}

\author{
Kelkkanen, Kari André; Lundqvist, Bengt; Nørskov, Jens Kehlet
}

Published in:

Journal of Chemical Physics

Link to article, DOI:

$10.1063 / 1.3193462$

Publication date:

2009

Document Version

Publisher's PDF, also known as Version of record

Link back to DTU Orbit

Citation (APA):

Kelkkanen, K. A., Lundqvist, B., \& Nørskov, J. K. (2009). Density functional for van der Waals forces accounts for hydrogen bond in benchmark set of water hexamers. Journal of Chemical Physics, 131(4), 046102. https://doi.org/10.1063/1.3193462

\section{General rights}

Copyright and moral rights for the publications made accessible in the public portal are retained by the authors and/or other copyright owners and it is a condition of accessing publications that users recognise and abide by the legal requirements associated with these rights.

- Users may download and print one copy of any publication from the public portal for the purpose of private study or research.

- You may not further distribute the material or use it for any profit-making activity or commercial gain

- You may freely distribute the URL identifying the publication in the public portal 


\title{
Density functional for van der Waals forces accounts for hydrogen bond in benchmark set of water hexamers
}

\author{
André K. Kelkkanen, ${ }^{1}$ Bengt I. Lundqvist, ${ }^{1,2, a)}$ and Jens K. Nørskov ${ }^{1}$ \\ ${ }^{1}$ Department of Physics, Center for Atomic-Scale Materials Design, Technical University of Denmark, \\ DK-2800 Kgs. Lyngby, Denmark \\ ${ }^{2}$ Department of Applied Physics, Chalmers University of Technology, SE-41296 Göteborg, Sweden
}

(Received 30 May 2009; accepted 11 July 2009; published online 29 July 2009)

[DOI: $10.1063 / 1.3193462]$

A recent extensive study ${ }^{1}$ investigates how various exchange-correlation (XC) functionals treat hydrogen bonds (HBs) in water hexamers, a critical HB benchmark set. Accurate wave-function studies including perturbative (MP2), ${ }^{1}$ quantum-Monte Carlo (DQMC), ${ }^{1}$ and coupled-cluster $[\operatorname{CCSD}(\mathrm{T})]$ (Ref. 2) calculations agree on the relative energetics of the low-lying isomers: ${ }^{1}$ The prism has lowest energy followed by (in order) cage, book, and cyclic isomers (Fig. 1). The study ${ }^{1}$ shows that traditional generalized gradient approximation (GGA) and hybrid functionals used in density-functional theory (DFT) give the wrong dissociationenergy trend of the four isomers (Fig. 1) and that van der Waals (vdW) dispersion forces give key contributions to the dissociation energy and asks whether functionals that incorporate $\mathrm{vdW}$ forces implicitly into the $\mathrm{XC}$ functional predict the correct trend and yield accurate total dissociation-energy values. ${ }^{1}$ Here an affirmative answer is given for the vdW-DF functional. ${ }^{3}$

Typically, a HB is an intermolecular bond between an intramolecular bond of an $\mathrm{H}$ atom on a donor molecule and an acceptor molecule. The directionality is the angle between these bonds. Studies on various HB-ed dimers suggest that $\mathrm{vdW}$ forces can have a significant effect on the strength of the $\mathrm{HB}$, including an indicated directionality effect. ${ }^{4}$ The DFT-local-density approximation (LDA) and DFT-GGAs also fail to describe highly bent HBs, another effect blamed on their lack of vdW account. ${ }^{5}$

For calculation of electronic structure and total energy, DFT offers an alternative to wave-function methods that is particularly powerful for extended systems, a well-proven excellent tool for strongly bound systems. For also abundant sparse systems that are bound by weak nonbonding forces, including HBs, nonlocal correlation functionals have been proposed recently. One such, the vdW-DF (Ref. 3) includes $\mathrm{vdW}$ forces in a seamless fashion with a derived XC functional,

$$
E_{\mathrm{vdW}-\mathrm{DF}}=E_{X}^{\mathrm{revPBE}}+E_{C}^{\mathrm{LDA}}+E_{C}^{\mathrm{nl}}
$$

where

$$
E_{C}^{\mathrm{nl}}=\frac{1}{2} \int d^{3} r d^{3} r^{\prime} n(\vec{r}) \phi\left(\vec{r}, \vec{r}^{\prime}\right) n\left(\vec{r}^{\prime}\right)
$$

depends nonlocally on the density $n(\vec{r})$ with a general kernel $\phi$ derived from first principles. ${ }^{3}$ Already used successfully for a wide spectrum of systems, ${ }^{6}$ its virtues, e.g., biological molecules, are highlighted by its account of stacking and HB interactions between nucleobases ${ }^{7}$ and DNA intercalation. ${ }^{8}$ The vdW-DF describes vdW effects approximately ${ }^{3,9}$ but differs significantly from another method doing so, DFT-D, ${ }^{10}$ being derived from first principles and thus nonempirical and informing about the electron structure.

All calculations are made with the GPAW software. ${ }^{11}$ The vdW-DF (Ref. 3) implementation is the same real-space method as in Ref. 14, which adds vdW forces perturbatively ${ }^{3}$ to a self-consistent revPBE ${ }^{15}$ calculation. The latter uses a well converged grid spacing of $0.13 \AA$ and Eq. (2) is evaluated on a grid twice as dense (a grid spacing of $0.15 \AA$ A results in binding-energy differences smaller than $1 \%$ ). The hexamer is in a cubic cell with side $18 \AA$ and 35 electronic bands are used. The dissociation energy is calculated as in Ref. 1 but with the vdW-DF.

Figure 1 shows trends in dissociation-energy values of the water-hexamer isomers relative to the prism configuration. Even within the narrow energy scale, the trends in the results from wave-function methods [MP2 (Ref. 1), DQMC (Ref. 1), and CCSD(T) (Ref. 2)] are very distinct and close to each other and to earlier $\operatorname{CCSD}(\mathrm{T})$ results. ${ }^{16}$ These trends cannot be accounted for by DFT with traditional GGA [PBE (Ref. 17), RPBE (Ref. 18), and revPBE (Ref. 15)] and hybrid [PBE0 (Ref. 13)] XC functionals. ${ }^{1}$ Clearly, the vdW-DF and DFT-D methods give both the right stable configuration and the proper energetic order of the lowest isomers.

To follow a trend is not a sufficient criterion for a good $\mathrm{XC}$ functional. For instance, LDA gives trend but is ignored due to lack of basic nonlocal interactions and its known overestimate of dissociation of water clusters by more than $50 \%$. ${ }^{1,19}$ The vdW-DF dissociation-energy values of the lowlying isomers show an underbinding of about $50 \mathrm{meV} / \mathrm{H}_{2} \mathrm{O}$ (Table I). At typical vdW separations (about 3-5 $\AA$ ) the accuracy of vdW-DF might be about $0.01 \mathrm{eV}^{14}$ but it is expected to be worse at the short separations of HBs (O-H separations being $1.9 \AA$ in the water dimer ${ }^{20}$ and, e.g., $1.7-2.0 \AA$ in the water hexamer cage, ${ }^{1}$ with vdW-DF and MP2 numbers given in Table I). The revPBE exchange functional ${ }^{15}$ used in vdW-DF lies close to exact exchange [Hartree-Fock (HF)] results for, e.g., argon, krypton, ${ }^{3}$ and benzene dimers ${ }^{21}$ but gets repulsive at shorter separations, 


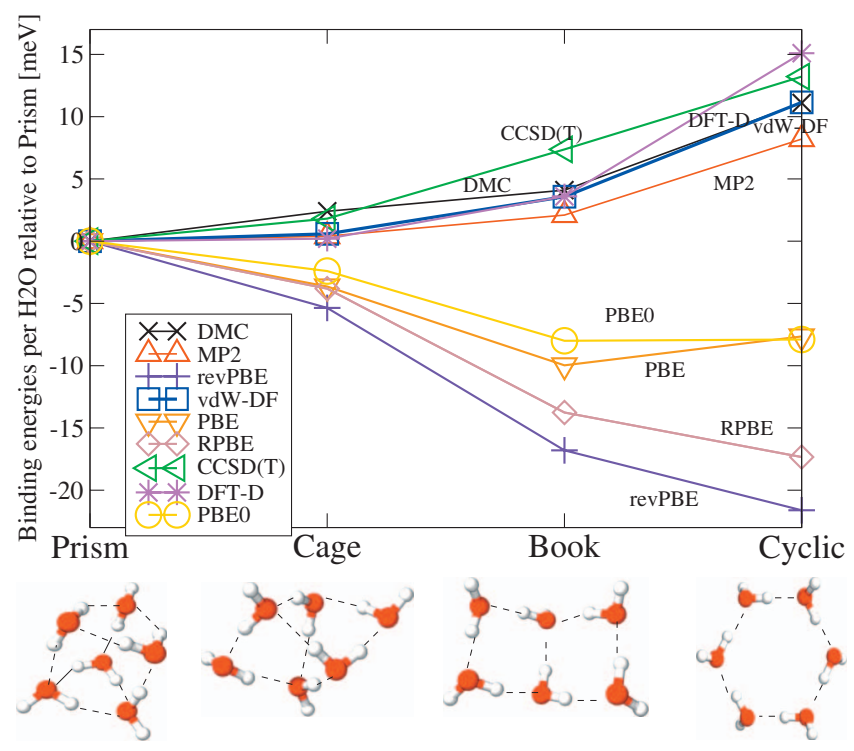

FIG. 1. Dissociation energy per $\mathrm{H}_{2} \mathrm{O}$ molecule of the water hexamer in low-lying isomers shown below calculated with wave-function [MP2 (Ref. 1), DQMC (Ref. 1), and CCSD(T) (Ref. 2)] and DFT methods [with XC functionals in the PBE, RPBE, and revPBE flavors of GGA and in PBE0 (Ref. 1), DFT-D (Ref. 1), and vdW-DF] relative to the prism isomer.

where other flavors of GGA might be closer as for the water dimer (Fig. 2). The relatively small underbinding by use of other less repulsive exchange functionals such as PBE, ${ }^{17} \mathrm{HF}$, and a hopefully soon developed systematically improved exchange functional. The latter should be accurate in regions where the HB applies and where vdW and covalent bonds have comparable strengths. Figure 2 shows that there is room for further stabilization for the hexamer. We believe that the relative error found for the water hexamer will translate to other more complex water clusters and that the vdW-DF could be expected to predict structure and phase diagrams of liquid water.

We thank Angelos Michaelides, Biswajit Santra, and Matthias Scheffler for providing the hexamer structures calculated in Ref. 1. The Center for Atomic-Scale Materials Design is funded by the Lundbeck Foundation.

TABLE I. Calculated dissociation-energy values in $m e V / \mathrm{H}_{2} \mathrm{O}$ and shortest separations between hydrogen and oxygen atoms $(\mathrm{R}-\mathrm{O}-\mathrm{H})$ of the low-lying water-hexamer isomers. Absolute-energy values $E_{\text {diss }}$ calculated with the vdW-DF (Ref. 3) allows comparison with MP2 result (Ref. 1) (numbers in parentheses), and relative energy values $E_{\text {reldiss }}$ with results from a recent $\operatorname{CCSD}(\mathrm{T})$ calculation (Ref. 2) (numbers in square brackets).

\begin{tabular}{lcccc}
\hline \hline & Prism & Cage & Book & Cyclic \\
\hline$E_{\text {diss }}$ & $-280(-332)$ & $-279(-332)$ & $-277(-330)$ & $-269(-324)$ \\
$E_{\text {reldiss }}$ & $0[0]$ & $1[2]$ & $4[7]$ & $11[13]$ \\
$\mathrm{R}-\mathrm{O}-\mathrm{H}(\AA)$ & $1.8(1.7)$ & $1.9(1.9)$ & $1.8(1.8)$ & $1.8(1.7)$ \\
\hline \hline
\end{tabular}

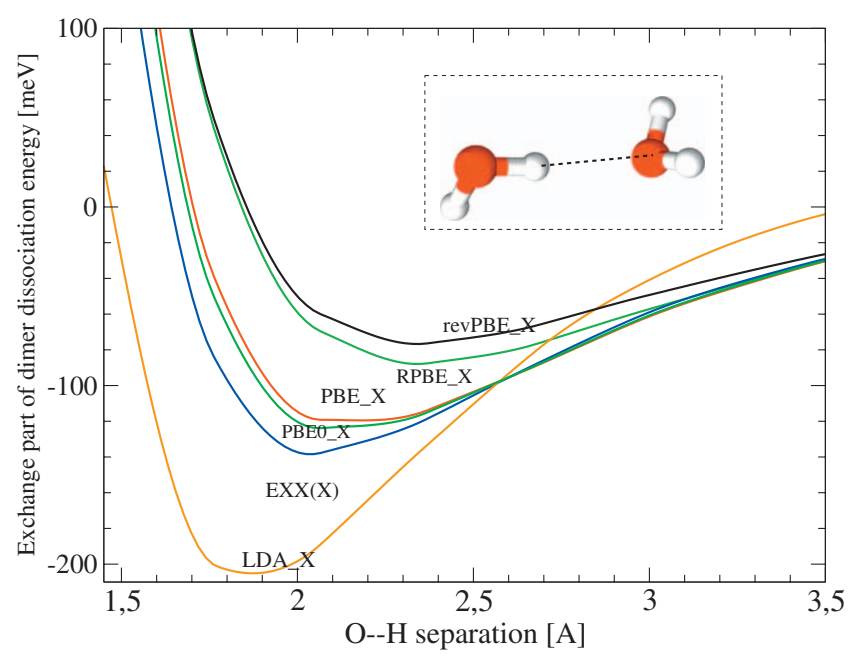

FIG. 2. Exchange part of the dissociation energy for the water dimer at varying separations (defined by inset) with exact exchange (HF) calculated by the $\operatorname{EXX}(\mathrm{X})$ method (Ref. 12) and with DFT using PBE, RPBE, revPBE, and LDA XC functionals, each calculated self-consistently and then subtracting the correlation energy. LDA exchange is showed to illustrate its tendency to overbind. The nonself-consistent (PBE densities) exchange part of PBE0 is also included by mixing of PBE and EXX exchange as in Ref. 13.

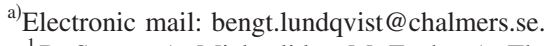

${ }^{1}$ B. Santra, A. Michaelides, M. Fuchs, A. Tkatchenko, C. Filippi, and M. Scheffler, J. Chem. Phys. 129, 194111 (2008).

${ }^{2}$ D. Bates and G. Tschumper, J. Phys. Chem. A 113, 3555 (2009).

${ }^{3}$ M. Dion, H. Rydberg, E. Schröder, D. C. Langreth, and B. I. Lundqvist, Phys. Rev. Lett. 92, 246401 (2004).

${ }^{4}$ T. Steiner and G. R. Desiraju, Chem. Commun. (Cambridge) 8, 891 (1998).

5 J. N. Ireta and M. Scheffler, J. Phys. Chem. A 108, 5692 (2004).

${ }^{6}$ D. C. Langreth, B. I. Lundqvist, S. D. Chakarova-Kack, V. R. Cooper, M. Dion, P. Hyldgaard, A. Kelkkanen, J. Kleis, L. Kong, S. Li, P. G. Moses, E. Murray, A. Puzder, H. Rydberg, E. Schröder, and T. Thonhauser, J. Phys.: Condens. Matter 21, 084203 (2009).

${ }^{7}$ V. R. Cooper, T. Thonhauser, A. Puzder, E. Schröder, B. I. Lundqvist, and D. C. Langreth, J. Am. Chem. Soc. 130, 1304 (2008); V. R. Cooper, T. Thonhauser, and D. C. Langreth, J. Chem. Phys. 128, 204102 (2008).

${ }^{8} \mathrm{~S}$. Li, V. R. Cooper, T. Thonhauser, B. I. Lundqvist, and D. C. Langreth, "Stacking interactions and DNA intercalation," J. Phys. Chem. B (in press).

${ }^{9}$ T. Thonhauser, V. R. Cooper, S. Li, A. Puzder, P. Hyldgaard, and D. C. Langreth, Phys. Rev. B 76, 125112 (2007).

${ }^{10}$ S. Grimme, J. Comput. Chem. 25, 1463 (2004).

${ }^{11}$ J. J. Mortensen, L. B. Hansen, and K. W. Jacobsen, Phys. Rev. B 71, 035109 (2005).

${ }^{12}$ M. Stadele, M. Moukara, J. Majewski, P. Vogl, and A. Görling, Phys. Rev. B 59, 10031 (1999).

${ }^{13}$ C. Adamo and V. Barone, J. Chem. Phys. 110, 6158 (1999).

${ }^{14}$ P. G. Moses, J. J. Mortensen, B. I. Lundqvist, and J. K. Nørskov, J. Chem. Phys. 130, 104709 (2009).

${ }^{15}$ Y. Zhang and W. Yang, Phys. Rev. Lett. 80, 890 (1998).

${ }^{16}$ R. M. Olson, J. L. Bentz, R. A. Kendall, M. W. Schmidt, and M. S. Gordon, J. Chem. Theory Comput. 3, 1312 (2007).

${ }^{17}$ J. P. Perdew, K. Burke, and M. Ernzerhof, Phys. Rev. Lett. 77, 3865 (1996).

${ }^{18}$ B. Hammer, L. B. Hansen, and J. K. Nørskov, Phys. Rev. B 59, 7413 (1999).

${ }^{19}$ C. Lee, H. Chen, and G. Fitzgerald, J. Chem. Phys. 101, 4472 (1994).

${ }^{20}$ T. Taketsugu and D. J. Wales, Mol. Phys. 100, 2793 (2002).

${ }^{21}$ A. Puzder, M. Dion, and D. C. Langreth, J. Chem. Phys. 124, 164105 (2006). 